\title{
Radiant Ignition of a Reactive Solid with In-Depth Absorption
}

\author{
A. LINAN \\ Escuela de Ingenieros Aeronáuticos, Ciudad Universitaria, Madrid, and \\ Instituto Nacional de Técnica Aerospacial "Esteban Terradas", Madrid, Spain \\ and \\ F. A. WILLIAMS \\ Department of the Aerospace and Mechanical Engineering Sciences, University of California, \\ San Diego, La Jolla, California
}

\begin{abstract}
An asymptotic analysis of the limit of large activation energy is presented for radiant ignition of a solid that experiences a one-step Arrhenius reaction in the condensed phase. Both constant and time-dependent radiant-energy fluxes are considered, and the complete range of values is covered for the absorption coefficient $\mu$. It is shown that as $\mu$ increases, the structure of the transition stage, which follows the inert heat-conduction stage, passes from a thermal explosion without heat conduction, to a single transient heat-conduction zone with distributed chemical heat release, to a two-zone structure composed of a reactive-diffusive-absorptive zone near the surface and a transient-diffusive zone in the interior. For very high values of $\mu$, the reactive-diffusive-absorptive zone further splits into a surface absorption zone and an interior reactive-diffusive zone, thereby reproducing results obtained previously for ignition by a surface-applied energy flux. The analysis shows that contrary to earlier expectation, the nondimensional absorption coefficient must be at least as large as the nondimensional activation energy for in-depth absorption to affect the ignition time negligibly.
\end{abstract}

\section{Introduction}

In a previous paper [1] we have employed asymptotic methods to investigate, in the limit of large activation energy, the ignition of a reactive solid by a constant energy flux applied to its surface. It has generally been assumed that during radiant ignition of solid propellants, the absorption layer is thin enough for the approximation of a surface-applied energy flux to be valid. However, a theoretical determination of conditions necessary for the neglect of in-depth absorption has not been reported. Our previous finding that a narrow layer at the surface is of critical importance during the transition stage preceding ignition, suggests that the traditional approximation may be questionable. Therefore we have performed the further analyses reported herein.

Difficulties in estimating the effect of in-depth absorption stem from two problems that arise in defining the absorption coefficient $\mu$. First, particularly for polymeric materials in the vicinity of absorption bands, $\mu$ may depend appreciably on the frequency of the incident radiation. Analyses neglecting this wavelength dependence will be most nearly applicable either for monochromatic laser radiation or for arc-image radiation applied to materials whose absorptivities vary but slightly over the energy-containing wavelength region of the arc. The principal part of the following development ignores any wavelength distribution. However, in the final section we shall show that concepts of superposition enable some conclusions to be drawn concerning ignition of materials with wavelength-dependent absorbtivities.

A second problem that is especially severe for heterogeneous materials, concerns the consequences of scattering of radiation. In a crude approximation, one may presume that for heterogeneities of sufficiently fine scale, the overall effect of scattering is to provide an additional diffuse contribution to the reflection coefficient and a modification to the effective value of $\mu$. The 
constant $\mu$ employed herein will be presumed to represent an effective overall absorption coefficient that includes, in an averaged sense, both scattering and a frequency distribution. The accuracy of this approximation has not been investigated.

These complications suggest that only careful measurements can produce useable values of $\mu$. Measurements have been made for polymers such as polystyrene and epoxy by Ohlemiller and Summerfield [2], who have seriously questioned the neglect of in-depth absorption [3] .

Their absorbtivities range from $74 \mathrm{~cm}^{-1}$ for pure polystyrene to $1200 \mathrm{~cm}^{-1}$ for material containing $20 \%$ carbon black. On the other hand, Evans, Beyer, and McCulley [4] report effective values of $\mu$ from $10^{3}-10^{5} \mathrm{~cm}^{-1}$ for pressed ammonium perchlorate powder, and they state that the values are higher if small amounts of catalyst are added. Certainly transparencies of different materials differ greatly. We estimate that in radiant ignition experiments effective values of $\mu$ may range from $10 \mathrm{~cm}^{-1}$ (e.g., for nonopacified double-base formulations) to $10^{5} \mathrm{~cm}^{-1}$, although these bounds in fact may be too narrow.

In the present work we employ, as closely as possible, the methods and notation of Ref. 1 . Therefore the thermal conductivity $\lambda$ and the thermal diffusivity $\kappa$ of the solid are assumed to be constant, and the initial temperature $T_{0}$ of the solid is taken to be uniform. The ratio of $\lambda T_{0}$ to the nonreflected portion $\dot{q}$ of the incident radiant energy flux at ignition will be employed as the characteristic length in forming the nondimensional spatial variable $\xi$. The quantity $\left(\lambda T_{0} / \dot{q}\right)^{2} / \kappa$ is the characteristic heat-conduction time that will be used to form the nondimensional time variable $\tau$. Dependent variables are $\theta(\xi, \tau) \equiv T / T_{0}$, where $T$ is the local instantaneous temperature, and $\epsilon(\xi, \tau)$, the ratio of the product concentration to the final product concentration. Nondimensional parameters that appeared in the previous paper are a Damköhler number $A$, defined as the ratio of the characteristic heat-conduction time to a characteristic chemical time, the latter being based solely on the pre-exponential rate factor; the nondimensional activation energy $E^{\prime} \equiv T_{a} / T_{0}$, where $T_{a}$ is the activation temperature, the order $a$ of the chemical reaction, and the heat-release parameter $B \equiv\left(T_{f}-T_{0}\right) / T_{0}$, where $T_{f}$ is the adiabatic flame temperature of the ignition reaction. The additional nondimensional parameter needed herein is the nondimensional absorption coefficient $\alpha \equiv \mu \lambda T_{0} / \dot{q}$.

The range of values of $\alpha$ that is of practical interest can be inferred by using the previously discussed range of values of $\mu$. Since solids with high absorbtivities often also have high thermal conductivities (e.g., materials containing high percentages of metalic constituents) and those with low values of $\mu$ tend to have low values of $\lambda$, the range of $\alpha$ exceeds that of $\mu$. Employing $T_{0}=$ $300^{\circ} \mathrm{K}, \lambda$ from $3 \times 10^{-4}-3 \times 10^{-2} \mathrm{cal} / \mathrm{cm}-\mathrm{sec}-{ }^{\circ} \mathrm{K}$ and $\dot{q}$ from $10-100 \mathrm{cal} / \mathrm{cm}^{2}-\mathrm{sec}$ we find that $10^{-2} \lesssim \alpha \lesssim 10^{4}$. Since generally $1 \ll E^{\prime} \leq 10^{2}$, it is clear that there is reason to study a range of values of $\alpha$ that includes the extremes $\alpha<1$ and $\alpha \gg E^{\prime}$. This complete range will be covered herein.

In the absence of an analysis such as that performed below, sufficient conditions for indepth absorption to be negligible cannot be stated. For example, Wise, Inami, and McCulley [5] used the condition $\alpha^{2} r>10$ as a justification for neglecting the influence of in-depth absorption on the ignition time. We shall show that a more precise condition is $\alpha \gtrsim E^{\prime}$. We shall also derive formulas that predict the extent to which the ignition time is increased for $\alpha<E^{\prime}$.

Section 2 briefly formulates the ignition problem. In Section 3, properties of the inert stage for constant flux with in-depth absorption are discussed. Next, the various regimes of $\alpha$ that arise will be indicated. The two major regimes are analysed separately in Sections 5 and 6 . The merging of these two regimes and the limit of thermal explosions are discussed in Section 7. Effects of time-varying flux, wavelength distributions and multidimensionality are described in Section 8.

Readers who are interested primarily in final results for ignition times under conditions of constant radiant flux may turn directly to Eq. 36 for $\alpha \gtrsim E^{\prime}$, to Eq. 51 for $\alpha \sim 1$, to Eq. 53 for $1<<\alpha<E^{\prime}$ or to Eq. 56 for $\alpha \ll 1$; alternatively, they may consult Fig. 3, which summarizes these 
results. Readers who are concerned with variable flux will find ignition-time formulas in Eqs. 57 and 58 and an appropriate plot in Fig. 4.

\section{Formulation}

Appropriate nondimensional parameters and variables have been defined in the introduction. In addition to these quantities, we introduce the function $I(t)$, which is defined as the ratio of the instantaneous incident radiant flux to the incident radiant flux at ignition. For clarity of presentation, we shall assume that $I(t)=1$, except in the final section, where the effect of an arbitrary flux history is ascertained. Gasification and surface regression are neglected throughout.

The mathematical problem that must be solved can be written as

$\theta_{\tau}=$

$\theta_{\xi \xi}+I \alpha \exp (-\alpha \xi)+A\left(1-\epsilon^{a} \exp \left(-\frac{E^{\prime}}{\theta}\right)\right.$

and

$$
\epsilon_{\tau}=\left(\frac{A}{B}\right)\left(1-\epsilon^{a} \exp \left(-\frac{E^{\prime}}{\theta}\right)\right.
$$

with the initial conditions

$$
\epsilon(\xi, 0)=0 \quad, \quad \theta(\xi, 0)=1
$$

and the boundary conditions

$$
\epsilon(\infty, \tau)=0 \quad, \quad \theta(\infty, \tau)=1
$$

and

$$
\theta \xi(0, \tau)=0 .
$$

In Eq. 1 the factor $e^{-\alpha \xi}$, which is equivalent to Beer's law, should provide an acceptable description of in-depth absorption of radiation, although in no sense is it an exact deduction from the relevant equation for transport of radiation. According to Eq. 5, heat loss from the surface is neglected; this should be a good approximation for solids igniting in gaseous atmospheres of moderate or low density.

\section{The Inert Stage}

We parallel our earlier work in seeking the value of $A$ that produces thermal runaway at a specified time $\tau_{c}$, which is selected consistent with the requirement that the corresponding increase in surface temperature under inert heating, $\theta_{c}-1$, is of order unity. This requirement is imposed to assure that there exists an inert heating stage prior to the events that lead to ignition and that ignition occurs before temperatures are reached that are too high for a well-defined ignition event to exist. It can be shown that unless $\theta_{c}-1 \gg 1 / E^{\prime}$, a distinct inert stage disappears, causing ignition to merge with the phenomenon of adiabatic thermal explosion, and unless $\theta_{c}-1 \ll E^{\prime}$, chemical energy will be released at too low a rate to produce significant departures from inert heating. In view of these bounds, the fact that $E^{\prime}$ seldom exceeds $10^{2}$ demonstrates that there is little physical interest in considering the order of $\theta_{c}-1$ to be other than unity.

In the inert stage that precedes transition to ignition, to lowest order, $\epsilon=0$, and $\theta=\theta_{I}(\xi, \tau)$, which for constant flux obeys the equation

$$
\theta_{I T}=\theta_{I} \xi \xi+\alpha \exp (-\alpha \xi),
$$

with the initial and boundary conditions

$$
\theta_{I}(\xi, 0)=\theta_{l}(\infty, \tau)=1, \quad \theta_{l \xi}(0, \tau)=0 .
$$

The solution to the problem defined by Eqs. 6 and 7 has been given by Boehringer and Spindler [6]; it is

$$
\theta_{I}=1+\alpha^{-1} f\left(\alpha \xi, \alpha^{2} \tau\right),
$$

where

$$
\begin{aligned}
& f(u, v) \equiv \\
& 2 \sqrt{\frac{v}{\pi}} \exp \left(-\frac{u^{2}}{4 v}\right)-u \operatorname{erfc}\left(\frac{u}{2 \sqrt{v}}\right)-e^{-u} \\
& +\frac{1}{2} e^{\nu}\left[e^{u} \operatorname{erfc}\left(\sqrt{v}+\frac{u}{2 \sqrt{v}}\right)\right. \\
& \left.+e^{-u} \operatorname{erfc}\left(\sqrt{v}-\frac{u}{2 \sqrt{v}}\right)\right]
\end{aligned}
$$


Equations 8 and 9 predict that the peak temperature always occurs at the surface of the solid. The expansion about the surface is

$$
\theta_{I} \simeq 1+2 \sqrt{\tau / \pi}-(1-g)\left(\alpha^{-1}+\alpha \xi^{2} / 2\right),
$$

where we have introduced the abbreviation

$$
g \equiv \exp \left(\alpha^{2} \tau\right) \operatorname{erfc}(\alpha \sqrt{\tau})
$$

since this particular function of $\alpha^{2} \tau$ appears repeatedly. The expansion of the preceding expression for $\theta_{l}$ about $\tau_{c}$ is

$$
\theta_{l} \simeq \theta_{c}+\alpha g_{c}\left(\tau-\tau_{c}\right)-\alpha\left(1-g_{c}\right) \xi^{2} / 2,
$$

where

$$
\theta_{c}=1+2 \sqrt{\tau_{c} / \pi}-\alpha^{-1}\left(1-g_{c}\right),
$$

and where $g_{c}$ denotes the function $g$ evaluated at $\alpha^{2} \tau=\alpha^{2} \tau_{c}$. For small values of $\alpha^{2} \tau_{c}$, Eq. 12 reduces to

$$
\theta_{c} \simeq 1+\alpha \tau_{c},
$$

and for large values of $\alpha^{2} \tau_{c}$ it becomes

$$
\theta_{c} \simeq 1+2 \sqrt{\tau_{c} / \pi}
$$

Equation 12 shows that if $\alpha$ is of order unity, then $\theta_{c}-1$ becomes of order unity for $\tau_{c}$ of order unity. Equation 13 implies that for $\alpha \ll 1$, $\theta_{c}-1$ will become of order unity for $\tau_{c}$ large, of order $\alpha^{-1} ;$ Eq. 13 is valid in this case because $\alpha^{2} \tau_{c}$ is small, of order $\alpha$. Equation 14 implies that for $\alpha \gg 1, \theta_{c}-1$ becomes of order unity for $\tau_{c}$ of order unity; in this case $\alpha^{2} \tau_{c}$ is large, of order $\alpha^{2}$, and so Eq. 14 is valid. Thus, we shall assume that $\tau_{c}$ is of order unity for $\alpha \geq 1$ and of order $\alpha^{-1}$ for $\alpha \ll 1$. From this observation, we can establish the bounds

$$
1 \ll A \ll \alpha\left[(1+\alpha) E^{\prime}\right]^{-1} \exp \left(E^{\prime}\right),
$$

which must be satisfied for the present analysis to be valid. The first condition assures that the chemical heat-release rate can become large enough compared with the radiant heating rate to cause thermal runaway (see Ref. 1), and the second that adiabatic thermal explosion with negligible preheating, which requires a time $\tau_{I}=\left(A E^{\prime}\right)^{-1} \exp \left(E^{\prime}\right)$, does not precede ignition. For small values of $\alpha^{2} \tau$, unless $\alpha \xi$ is small compared with unity, Eqs. 8 and 9 reduce to

$$
\theta_{l} \simeq 1+\alpha \tau e^{-\alpha \xi}
$$

which simply represents absorption with negligible heat conduction; there is a layer at the surface in which the order $\xi$ does not exceed the order of $\sqrt{\tau}$, where transient heat conduction is of importance. For large values of $\alpha^{2} t$, unless $\alpha \xi$ is large compared with unity, Eqs. 8 and 9 reduce to

$$
\theta_{I} \simeq 1+2 \sqrt{\tau / \pi}-\xi-\alpha^{-1} e^{-\alpha \xi} \text {, }
$$

which describes absorption and heat conduction with a negligible transient term in Eq. (6); for $\xi$ large compared with the thickness $\alpha^{-1}$ of the absorption layer, e.g., for $\xi$ of the order of $\sqrt{\tau}$, transient heat conduction becomes important and absorption negligible. Only in this limit of large $\alpha^{2} \tau$ does one retrieve the inert problem of Ref. 1; specifically, an expansion in the artificial small parameter $\alpha^{-1}$ produces an inner problem whose solution is

$$
\theta_{I}=\theta_{I}(0, \tau)-\xi-\alpha^{-1}\left(e^{-\alpha \xi}-1\right)
$$

and an outer problem described by the transient heat-conduction equation with initial and boundary conditions $\theta_{I}(\xi, 0)=\theta_{I}(\infty, \tau)=1$, $\theta_{I} \xi(0, \tau)=-1$, the last condition being obtained from matching with the first term of the inner expansion.

\section{Establishment of $\alpha$ Regimes}

it is clear from the preceding discussion that under the conditions of the problem analyzed in the previous paper, the thickness of the absorption layer is of order $\alpha^{-1}$. Since the thickness of the reaction layer was found in this case to be of order $1 / E^{\prime}$, it follows that the previous analysis is applicable only if $\alpha \gg E^{\prime}$.

When $\alpha$ becomes comparable with $E^{\prime}$, during the 
transition stage a surface layer will develop in which reaction and heat conduction occur in a region whose temperature field is influenced by in-depth absorption, and a new analysis will be needed. Such an analysis is given in the following section.

Typical values of $E^{\prime}$ are such that when $\alpha$ becomes small compared with $E^{\prime}$, the next interesting case occurs for $\alpha$ of order unity. In this case $\tau_{c}$ is of order unity, and therefore $\alpha^{2} \tau_{c}$ is not large. It follows that the inert problem of the previous paper is not applicable here and Eqs. 11 and 12 must be considered in their entirety. An analysis of the transition stage for this case will be given in Section 6, where it will be found that a single zone of transient heat conduction and distributed chemical reaction develops, instead of the two-zone structure obtained in the previous paper and in Section 5.

It will be seen in Section 7 that the results of Section 6 for large $\alpha$ merge with those of Section 5 for small $\alpha / E^{\prime}$ and that the results of Section 6 for small $\alpha$ describe a thermal explosion without heat conduction which occurs in a heated layer of thickness $\alpha^{-3 / 4} / \sqrt{E^{\prime}}$. This explosion possesses an ignition time that agrees somewhat curiously with a result obtained quite easily by using Eq. 1 with the heat-conduction term deleted entirely, even though the latter analysis would produce Eq. 16 and thereby ignore the conduction layer that exists at the surface for small $\alpha$ during the inert stage.

From the present discussion it is apparent that the results which will be obtained in Sections 5, 6, and 7 will cover the complete range of values of $\alpha$ that is of physical interest.

\section{Transition Stage for $\alpha$ and}

\section{$E^{\prime}$ of the Same Order}

When the order of $\alpha$ is the same as that of $E^{\prime}$, we write $\alpha=\alpha_{1} / \delta^{2}$, where $\delta \equiv \sqrt{\theta_{c}^{2} / E^{\prime}}$, and assume that $\alpha_{1}$ is of order unity. As in our previous paper, we expect to find a transition stage in which the appropriate time variable is

$$
\sigma=\left(\frac{E^{\prime}}{\theta_{c}^{2}}\right)\left(\tau-\tau_{c}\right)\left(\pi \tau_{c}\right)^{-1 / 2}+b_{0}^{\prime},
$$

where $b_{0}^{\prime}$, which we assume to be of order unity, is the first term of an expansion

$$
b=b_{0}^{\prime}+\delta b_{1}^{\prime}+\cdots
$$

of the quantity

$$
b \equiv \ln \left[\left(\pi r_{c}\right)^{1 / 4}\left(\frac{\theta_{c}^{2}}{E^{\prime}}\right)^{1 / 2} A \exp \left(\frac{-E^{\prime}}{\theta_{c}}\right)\right]
$$

Since $\alpha$ is large, in these expressions $\theta_{c}$ will be given by Eq. 14. Thermal runaway occurs at $\sigma=b_{0}^{\prime}$ to all algebraic orders in $\delta$, and the higher coefficients, $b_{1}^{\prime}, \ldots$, in Eq. 19 must be chosen to minimize the severity of the singularity at $\sigma=b_{0}^{\prime}$. Such a prescription is quite reasonable on physical grounds, since its effect is to extend the validity of the expansion procedure to as late a time as possible.

We anticipate a two-zone structure, with the thickness of the inner zone, in which absorption, heat conduction and chemical heat release occur, of order $1 / E^{\prime}$, and with the thickness of the outer zone of transient heat conduction, where the chemically liberated heat is distributed, of order $1 / \sqrt{E^{\prime}}$. As in our previous paper, both zones are thin compared with the width of the inert-stage transient heat-conduction region, whose thickness is of order unity in $\xi$. Thus, we introduce the inner spatial variable

$$
x=\left(\frac{E^{\prime}}{\theta_{c}^{2}}\right) \xi
$$

and the outer spatial variable

$$
\eta=\left(\pi \tau_{c}\right)^{-1 / 4}\left(\frac{E^{\prime}}{\theta_{c}^{2}}\right)^{1 / 2} \xi=\left(\pi \tau_{c}\right)^{-1 / 4}\left(\frac{\theta_{c}^{2}}{E^{\prime}}\right)^{1 / 2} x .
$$

Expansions for $\theta-\theta_{I}$ will be sought in the forms

$$
\theta-\theta_{l}=\delta^{2} \psi_{0}(x, \sigma)+\delta^{3} \psi_{1}(x, \sigma)+\cdots
$$

and

$$
\theta-\theta_{I}=\delta^{2} \Phi_{0}(\eta, \sigma)+\delta^{3} \Phi_{1}(\eta, \sigma)+\cdots,
$$


for the inner and outer zones, respectively. In the inner zone the expansion for $\epsilon$ is

$$
\epsilon=\delta \epsilon_{1}(x, \sigma)+\delta^{2} \epsilon_{2}(x, \sigma)+\cdots,
$$

and in the outer zone $\epsilon$ will be found to be exponentially small. When expanded in powers of $\delta$. Eqs. 8 and 9 give

$$
\begin{aligned}
\theta_{I} & =\theta_{c}+\delta^{2}\left[\sigma-b_{0}^{\prime}-x-\alpha_{1}^{-1} \exp \left(-\alpha_{1} x\right)\right] \\
& +O\left\{\delta^{x}\right\}
\end{aligned}
$$

in the inner variable and

$$
\theta_{I}=\theta_{c}-\left(\pi \tau_{c}\right)^{1 / 4} \delta \eta+O\left\{\delta^{2}\right\}
$$

in the outer variable.

Substitution of Eqs. 18-21, 23, 25 and 26 into Eqs. 1 and 2 produces the inner equations

$$
\begin{aligned}
\psi_{0 x \boldsymbol{x}} & +\delta \psi_{1 \boldsymbol{x} \boldsymbol{x}}=-\delta\left(\pi \tau_{c}\right)^{-1 / 4} \exp \left[\psi_{0}+\sigma-x\right. \\
& \left.-\alpha_{1}^{-1} \exp \left(-\alpha_{1} x\right)\right]
\end{aligned}
$$

up to terms of order $\delta^{2}$, and

$$
\begin{aligned}
\epsilon_{1 \sigma} & =B^{-1}\left(\pi \tau_{c}\right)^{1 / 4} \exp \left[\psi_{0}+\sigma-x\right. \\
& \left.-\alpha_{1}^{-1} \exp \left(-\alpha_{1} x\right)\right]
\end{aligned}
$$

up to terms of order $\delta$. From the term of order unity in Eq. 28 and the boundary condition for $\psi_{0}$ implied by Eq. 5, we conclude that $\psi_{0}$ depends only on $\sigma$. Equation 29 and the initial condition for $\epsilon$ implied by Eq. 3 then yield

$$
\begin{aligned}
\epsilon_{1} & =B^{-1}\left(\pi \tau_{c}\right)^{1 / 4} \exp [-x \\
& \left.-\alpha_{1}^{-1} \exp \left(-\alpha_{1} x\right)\right] \int_{-\infty}^{\sigma} \exp \left(\psi_{0}+\sigma\right) d \sigma .
\end{aligned}
$$

The terms of order $\delta$ in Eq. 28, along with the boundary condition $\psi_{\mathrm{I} \boldsymbol{x}}(0, \sigma)=0$, implied by Eq. 5, then yield

$$
\begin{aligned}
\psi_{1 x} & =-\left(\pi \tau_{c}\right)^{-1 / 4} \exp \left(\psi_{0}\right. \\
& +\sigma) \int_{0}^{x} \exp \left[-x-\alpha_{1}^{-1} \exp \left(-\alpha_{1} x\right)\right] d x
\end{aligned}
$$

It follows that

$$
\psi_{1 x}(\infty, \sigma)=-\left(\pi \tau_{c}\right)^{-1 / 4} G \exp \left(\psi_{0}+\sigma\right)
$$

where $G$ is a function of $\alpha_{1}$, defined by

$$
G \equiv \int_{0}^{\infty} \exp \left[-x-\alpha_{1}^{-1} \exp \left(-\alpha_{1} x\right)\right] d x
$$

It is easy to show from Eqs. 1, 2, 18-20, 22, 24, and 27 that the first term of the outer expansion of $\theta$ obeys the equation of transient heat conduction. The initial condition obtained from Eq. 3 is $\Phi_{0}(\eta,-\infty)=0$, and the boundary condition im. plied by Eq. 4 is $\Phi_{0}(\infty, \sigma)=0$. Matching to the inner expansion requires $\Phi_{0}(0, \sigma)=\psi_{0}$ and

$$
\Phi_{0 \eta}(0, \sigma)=-G \exp \left(\psi_{0}+\sigma\right)
$$

where use has been made of Eq. (32). The small time translation $\sigma^{\prime}=\sigma+\ln G$ then produces the problem

$$
\left.\begin{array}{r}
\Phi_{0 \sigma^{\prime}}=\Phi_{0 \eta \eta}, \Phi_{0}(\eta,-\infty)=\Phi_{0}\left(\infty, \sigma^{\prime}\right)=0, \\
\Phi_{0 \eta}\left(0, \sigma^{\prime}\right)=-\exp \left[\Phi_{0}\left(0, \sigma^{\prime}\right)+\sigma^{\prime}\right],
\end{array}\right\}
$$

which has been solved previously in Ref. 1 . Thermal runaway was found to occur at $\sigma^{\prime}=-0.431=b_{0}$. It follows that in the present problem

$$
b_{0}^{\prime}=b_{0}-\ln G .
$$

Equations 19-20 then show that, to lowest order in $\delta$,

$$
A \exp \left(-\frac{E^{\prime}}{\theta_{c}}\right)=\left(\frac{E^{\prime}}{\theta_{c}^{2}}\right)^{1 / 2}\left(\pi \tau_{c}\right)^{-1 / 4} G^{-1} \exp \left(b_{0}\right) .
$$

This expression for $A$ differs from that obtained previously only in the presence of the factor $G$. It can be shown that $G$ can be expressed in terms of the incomplete Gamma function as

$$
G=\alpha_{1}^{-1} \Gamma\left(\alpha_{1}^{-1}\right) \gamma^{*}\left(\alpha_{1}^{-1}, \alpha_{1}^{-1}\right),
$$


where $y^{*}$ is a single-valued analytic function of both of its arguments. Tables and graphs of $\Gamma$ and of $\gamma^{*}$ exist [7]. However, to obtain accurate palues of $G$, it is better to perform numerically the integration in Eq. 33 than to use the published results. We have calculated $G$ as a function of $\alpha_{1}$ by numerical integration and show the results in Fig. 1. Also shown in Fig. 1 is $A\left(\pi \tau_{c}\right)^{1 / 4} \alpha^{-1 / 2} \exp \left(-E^{\prime} / \theta_{c}\right)$ as a function of $a_{1}$, according to Eq. 36 . By using this last curve and Eq. 14 for $\theta_{c}$, the nondimensional rate constant $A$ can be obtained if $\alpha, E^{\prime}$ and $I_{c}$ are known.

As $\alpha_{1} \rightarrow \infty, G$ approaches unity, and we recover the result of our previous paper. An analytical expansion for large values of $\alpha_{1}$, obtained by using Eq. 33, is shown in Fig. 1, from which it can be seen that the expansion is quite accurate for $\alpha_{1} \geq 1$. For $\alpha_{1}$ of order unity, $G$ is of order unity, and Eqs. 14 and 26 show that approximately $\partial \tau_{c} / \partial G=-\sqrt{\pi \tau_{c}} \theta_{c}^{2} / E^{\prime} G$, in which all factors are of order unity except $E^{\prime}$, which is large. Therefore for $\alpha_{1}$ of order unity, the ignition time depends only weakly on $G$, and very little difference will be found between the ignition time obtained from Eq. 36 and that derived in the previous paper. On the other hand, for low values of $\alpha_{1}$, we obtain from Eq. 33

$$
\ln G \simeq-\alpha_{1}^{-1}+\ln \left[\sqrt{\alpha_{1}^{-1} \pi / 2}+\frac{1}{3}+O\left(\sqrt{\alpha_{1}}\right)\right] \text {, }
$$

which becomes large in magnitude and thereby produces a result which differs appreciably from that of our previous work. It can be seen from Fig. 1 that for $\alpha_{1} \leq 1$, the numerical results agree quite well with the asymptotic expression given by Eq. 38. As $\alpha_{1}$ becomes small, $\alpha$ tends to become of order unity, and the analysis given in the following section becomes appropriate.

\section{Transition Stage for $\alpha$ of Order Unity}

To analyze the transition stage for $\alpha$ of order unity, we follow a procedure analogous to that of the preceding section, except in that we introduce only one new spatial variable, since a single zone occurs. The appropriate time variable for the transition stage becomes

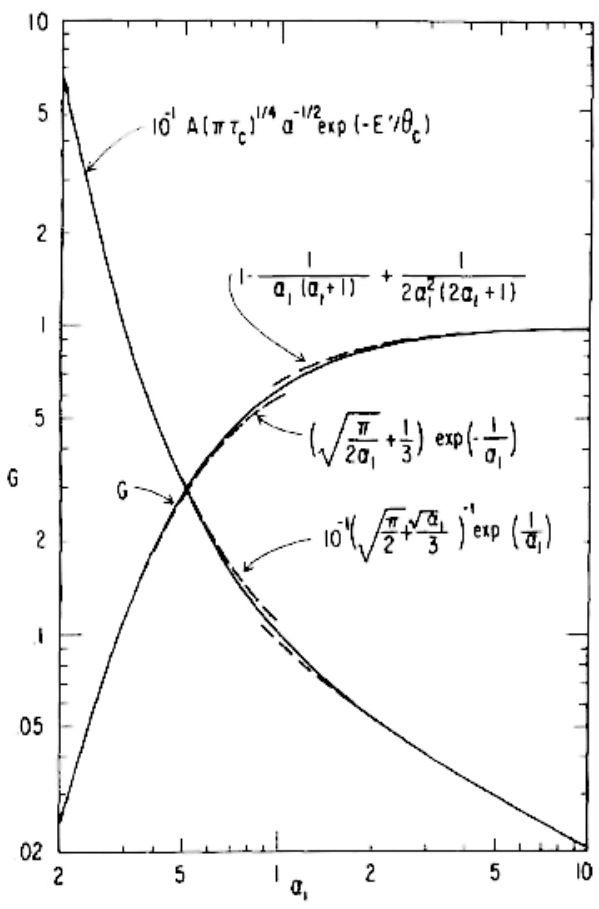

Fig. 1. $G$ and the nondimensional rate constant for ignition, as function of $\alpha \theta_{c}^{2} / E^{\prime}$, for $\alpha$ of order $E^{\prime}$.

$$
t=\left(\frac{E^{\prime}}{\theta_{c}^{2}}\right) \alpha g_{c}\left(\tau-\tau_{c}\right)+c_{0},
$$

where $c_{0}$, which is of order unity, is the first term of the expansion

$$
c=c_{0}+\delta^{2} c_{1}+\cdots,
$$

of the quantity

$$
c \equiv \ln \left[\alpha^{-1} g_{c}^{-1} A \exp \left(-E^{\prime} / \theta_{c}\right)\right],
$$

in which $\theta_{c}$ is given by Eq. 12. Thermal runaway occurs at $t=c_{0}$ to all algebraic orders in $\delta$, and $c_{1}, c_{2}, \ldots$ are to be chosen to minimize the order of the singularity at $t=c_{0}$. The thickness of the zone in which thermal runaway occurs is defined by $y$ of order unity, where 


$$
y=\left(\frac{E^{\prime}}{\theta_{c}^{2}}\right)^{1 / 2}\left(\alpha g_{c}\right)^{1 / 2} \xi .
$$

From this equation and Eq. 10 it is seen that the thickness of the runaway zone in $\xi$ is of order $1 / \sqrt{E^{\prime}}$ when $\alpha$ is of order unity or larger, and larger when $\alpha$ is small.

An expansion for $\theta-\theta_{I}$ will be sought in the form

$$
\theta-\theta_{I}=\delta^{2} \chi_{0}(y, t)+\delta^{4} \chi_{1}(y, t)+\cdots,
$$

and for $\epsilon$ in the form

$$
\epsilon=\delta^{2} \omega_{1}(y, t)+\delta^{4} \omega_{2}(y, t)+\cdots \cdot
$$

The relevant expansion of $\theta_{I}$, obtained from Eq. 11 , is

$$
\theta_{l}=\theta_{c}+\delta^{2}\left(t-c_{0}-F y^{2}\right)+O\left\{\delta^{4}\right\},
$$

where

$$
F \equiv \frac{\left(1-g_{c}\right)}{2 g_{c}}
$$

Substitution of Eqs. $39-45$ into Eqs. 1 and 2 yields, up to terms of order $\delta^{2}$,

$$
\chi_{0 t}-\chi_{0 y y}=\exp \left(\chi_{0}+t-F y^{2}\right)
$$

and

$$
\omega_{1 t}=B^{-1} \exp \left(X_{0}+t-F y^{2}\right)
$$

The solution to Eq. 48, consistent with the initial condition implied by Eq. 3, is

$$
\omega_{1}=B^{-1} \exp \left(-F y^{2}\right) \int_{-\infty}^{t} \exp \left[\chi_{0}(y, t)+t\right] d t
$$

which can be evaluated only after $\chi_{0}(y, t)$ is found. Equation 47 for $\chi_{0}$ is the heat conduction equation with a distributed nonlinear heat source; the initial and boundary conditions for this equa- tion, derived from Eqs. 3-5, are

$\chi_{0}(\infty, t)=\chi_{0}(y,-\infty)=\chi_{0 y}(0, t)=0$.

The problems for $\chi_{0}$, given by Eqs. 47 and 50 , is well set, and there is only one parameter, $F$, but the solution must be obtained numerically. By analogy with Eq. 34, it is clear that the solution will exhibit thermal runaway, at time $t=c_{0}$, which will depend on $F$ and therefore on $\alpha^{2} \tau_{c}$. If $\alpha^{2} \tau_{c}$ is of order unity, then $F$ and $c_{0}$ will be of order unity. From Eqs. 40 and 41, the formula for $A$, to lowest order in $\delta^{2}$, becomes

$$
A \exp \left(-\frac{E^{\prime}}{\theta_{c}}\right)=\alpha g_{c} \exp \left(c_{0}\right),
$$

in which $g_{c}$ is related to $\alpha^{2} \tau_{c}$ according to Eq. 10 . Equation 47 has been integrated numerically by a Crank-Nicholson implicit scheme employing quasilinearization of the source term. For large negative value of $t$, the solution is

$$
\begin{aligned}
\chi_{0} & =\frac{\sqrt{\pi}}{4 \sqrt{F}} e^{1 /(4 F)} e^{t}\left[e^{y} \operatorname{erfc}\left(\frac{1}{2 \sqrt{F}}+\sqrt{F y}\right)\right. \\
& \left.+e^{-y} \operatorname{erfc}\left(\frac{1}{2 \sqrt{F}}-\sqrt{F} y\right)\right]
\end{aligned}
$$

In performing the numerical integration for other values of $t$, the quantities $e^{t}$ and $\operatorname{erf}(y)$ were employed as independent variables, to produce convenient step sizes and finite boundary loca. tions. By completing the numerical integration for various values of $F$, the dependence of $c_{0}$ on $F$ was obtained. Use of this result in conjunction with Eqs. 10 and 46 provides $c_{0}$ as a function of $\alpha^{2} \tau_{c}$. From this result and Eq. 10, the quantity $(A / \alpha) \exp \left(-E^{\prime} / \theta_{c}\right)$ can be calculated as a func. tion of $\alpha^{2} \tau_{c}$ by means of Eq. 51. Equation 1 ? shows that $\alpha\left(\theta_{c}-1\right)$ is also a function of $\alpha^{2} r_{c}$. These various functions are plotted in Fig. 2.

A graph of $\tau_{c}$, as a function of $A$, for variou values of $\alpha$ and $E^{\prime}$, is shown in Fig. 3. The curva in Fig. 3 labeled $\log \alpha=\infty$ have been given earlie by Bradley [8] and are in agreement with Eq. 36, while those labeled $\log \alpha=-\infty$ are limiting lina 


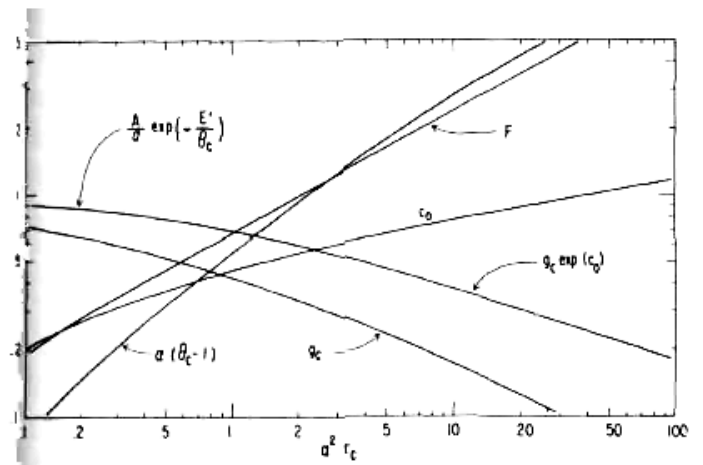

Fg. 2. $c_{0}, F, g_{c}$, nondimensional ignition temperature, and nondimensional rate constant, as function of nondimensional ignition time, for $\alpha$ of order unity.

that correspond to the adiabatic thermal-explosion time defined after Eq. 15. Figure 2 was used for ealculating all other curves shown in Fig. 3, except those with $\log \alpha=-2$ and -4 , for which the simpler limiting formula given in Eq. 56 was employed. From Fig. 3 it can be inferred that for given values of $\boldsymbol{A}$ and $E^{\prime}$, the ignition time obtained from Eq. 51 or from Fig. 2 is appreciably longer than that corresponding to Eq. 36. This is primarily a consequence of the fact that the time required to reach any given value of $\theta_{c}$ according to Eq. 12 is longer than that required according to Eq. 14, when $\alpha$ is of order unity or smaller. Since the expansion parameter here is $\delta^{2}$ instead of $\delta$ as in the previous work, for typical values of $E^{\prime}$ we expect the error in the value of $\boldsymbol{A}$ given by Eq. 51 to be less than $5 \%$ instead of the $20 \%$ error expected from Eq. 36 .

\section{Limiting Cases}

Further insight into the character of the dependence of ignition time upon $\alpha$ can be gleaned by investigating limiting cases of the previous analyses.

Consider first the form taken by Eq. 47 for large values of $\alpha$. From Eqs. 10 and 46 it follows that $F \rightarrow \alpha \sqrt{\pi \tau_{c}} / 2$ as $\alpha \rightarrow \infty$, and therefore the coefficient of $-y^{2}$ in the exponential in Eq. 47 becomes large. It follows that for $y$ of order unity the source term in Eq. 47 is negligible; an inner layer develops, where $y$ is of order $F^{-1 / 2}$, in which the source term must be considered. In this inner zone, the time derivative becomes small, and a

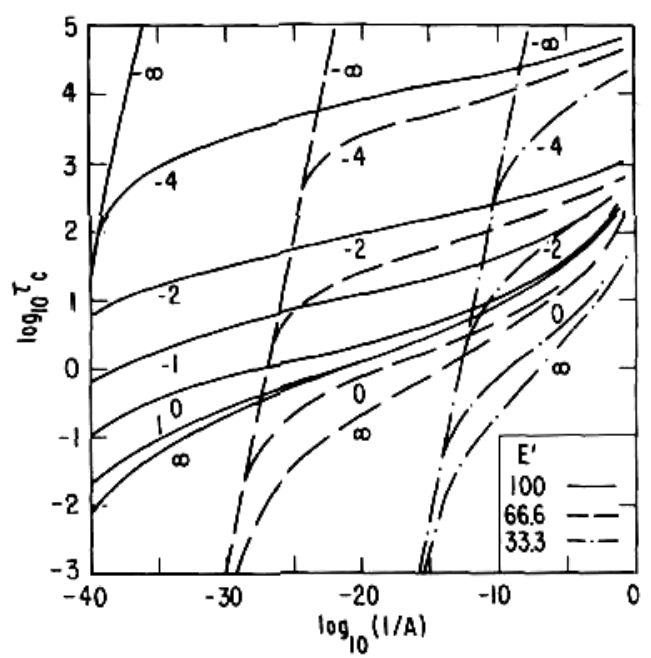

Fig. 3. Nondimensional ignition time as a function of nondimensional rate constant for various values of the nondimensional activation energy and absorbtivity. The numbers that label the curves are $\log _{10} \alpha$.

problem emerges that resembles Eq. 28, with the exception that the $x$ dependence inside the exponential becomes proportional to $-x^{2}$ instead of $-x-\alpha_{1}^{-1} \exp \left(-\alpha_{1} x\right)$. However, in the limit $\alpha_{1} \rightarrow 0$, this last quantity becomes $-\alpha_{1}^{-1}-\alpha_{1} x^{2} / 2$, which, with $\alpha_{1}^{-1}$ absorbed into a time translation, is of the same general form as $-x^{2}$. The respective length scales for the inner zones become the same, both being $\sqrt{2 \theta_{c}^{2} / \alpha E^{\prime}}$ in the $\xi$ coordinate. In addition, from Eq. 42 it can be seen that the length scale for the outer transient heat-conduction zone obtained from Eq. 47 is $\left(\pi \tau_{c}\right)^{1 / 4}\left(\theta_{c}^{2} / E^{\prime}\right)^{1 / 2}$, which is in agreement with the length scale for the problem defined by Eq. 34 , as can be seen from the definition of $\eta$ given in Eq. 22. Finally, we note that Eqs. 36 and 51 become identical in the limits $\alpha_{1} \rightarrow 0, \alpha \rightarrow \infty$, both reducing to

$$
\begin{aligned}
A \exp & {\left[-\left(\frac{E^{\prime}}{\theta_{c}}\right)\left(1+\frac{1}{\alpha \theta_{c}}\right)\right] } \\
& =\left(\pi \tau_{c}\right)^{-1 / 2}\left(2 \alpha{\sqrt{\tau_{c} / \pi}}^{1 / 2} \exp \left(b_{0}\right),\right.
\end{aligned}
$$

since the solution to the two-zone problem derived from Eq. 47 reveals that $c_{0} \rightarrow b_{0}+$ $\ln \left(2 \alpha{\left.\sqrt{\tau_{c} / \pi}\right)^{1 / 2}}^{1 /}\right.$ the limit. In Eq. 53, 
Eq. 14 is to be used for $\theta_{c}$. From these observations, it becomes clear that the results of Section 5 for small values of $\alpha_{1}$ agree with those of Section 6 for large values of $\alpha$ and that therefore solutions are now available which extend from $\alpha$ of order unity to $\alpha$ large compared with $E^{\prime}$.

Consider next the form taken by Eq. 47 for small values of $\alpha$. Since Eqs. 10 and 46 show that $F \rightarrow \alpha \sqrt{\tau_{c} / \pi}$ as $\alpha \sqrt{\tau_{c}} \rightarrow 0$, and since we have seen that $\alpha \sqrt{\tau_{c}} \rightarrow 0$ as $\alpha \rightarrow 0$, it follows that the coefficient of $-y^{2}$ in the exponential in Eq. 47 approaches zero as $\alpha \rightarrow 0$. Therefore the source strength changes negligibly across a layer whose thickness $\left(\theta_{c}^{2} / E^{\prime} \alpha\right)^{1 / 2}$ is such that $y$ is of order unity. To obtain a region in which the source changes appreciably, we must employ a spatial variable with the larger characteristic thickness $\left(\pi / \tau_{c}\right)^{1 / 4}\left(\theta_{c}^{2} / E^{\prime}\right)^{1 / 2} / \alpha$. In this variable, the space derivative in Eq. 47 is negligible in comparison with the time derivative, and we obtain a simple thermal explosion with negligible heat conduction, viz., $\chi_{0 t}=\exp \left(\chi_{0}+t-F y^{2}\right)$, whose solution

$$
\chi_{0}=-\ln \left(1-e^{t-F y^{2}}\right),
$$

first diverges at $t=0$ for $\boldsymbol{y}=0$.

Equation 54 satisfies all of the initial and boundary conditions given in Eq. 50, as well as Eq. 47 , provided that heat conduction is negligible. It implies that in the limit of very low absorbtivity $c_{0}=0$. A more formal calculation reveals that

$$
c_{0} \simeq 2 F-2+\frac{4}{3} \pi^{2} F^{2}+O\left(F^{3}\right)
$$

as $F$ approaches zero. Equation 55 can be used in conjunction with Eqs. 10, 46, and 51 to provide an expression for $\boldsymbol{A}$ that is valid for small values of $\alpha$. When use is made of Eq. 13 for $\theta_{c}$, it is found that the limiting form of this expression for very small values of $\alpha$ can be reduced to

$$
r_{c}=\left[E^{\prime} / \ln (A / \alpha)-1\right] / \alpha .
$$

This same result can be derived by using only Eqs. 1,3 , and 4 , and by neglecting heat conduction throughout; such an analysis produces Eq. 16 for the inert stage. In physical variables, Eq. 56 corre- sponds approximately to an ignition time that is inversely proportional to the first power, instead of the square, of the incident radiant flux. These results extend the range of $\alpha$, for which ignitiontime formulas are available, to values that are small compared with unity.

\section{Generalizations and Discussion}

In principle there is no difficulty in extending the results to arbitrary histories of radiant flux as a function of time, the only restriction being that the time rate of change of flux not be exceedingly large at the time of ignition. Since the transition stage is short compared with the inert stage, time-varying flux does not affect the structure of the transition stage, provided that in the time variable of the transition stage the logarithmic derivative of the flux is small. In the appendix, we presume that this condition is satisfied and indicate the changes that must be made in the preceding analyses to account for arbitrary flux. time histories. The results demonstrate that the only new problem requiring solution is the inertheating problem whose solution is expressed by Eq. 8. From the new function $f(u, v)$ that corresponds to the modified inert-heating problem, the quantities $\theta_{c}$ and $\theta_{c}^{\prime} \equiv \theta_{I \tau}\left(0, \tau_{c}\right)$ will both be expressible in terms of $\tau_{c}$. It can then be shown (see Appendix) that the appropriate generalizations of Eqs. 36 and 51, respectively, to conditions of arbitrary flux-time history, are

$$
A \exp \left(-\frac{E^{\prime}}{\theta_{c}}\right)=\left(\frac{E^{\prime} \theta_{c}^{\prime}}{\theta_{c}^{2}}\right)^{1 / 2} G^{-1} \exp \left(b_{0}\right)
$$

and

$$
A \exp \left(-\frac{E^{\prime}}{\theta_{c}}\right)=\theta_{c}^{\prime} \exp \left(c_{0}\right)
$$

with $c_{0}$ in the last expression related to the new $F$ defined in Eq. (A4) by Fig. 2. If $g_{c}$ is interpreted as $\theta_{c}^{\prime} / \alpha$ and the horizontal scale is interpreted as an arbitrary parameter that is not related to $\alpha^{2} \tau_{c}$, then all of the curves in Fig. 2, except for $\alpha\left(\theta_{c}-1\right)$, remain valid for arbitrary flux-time histories.

Because of its more general applicability, a graph 


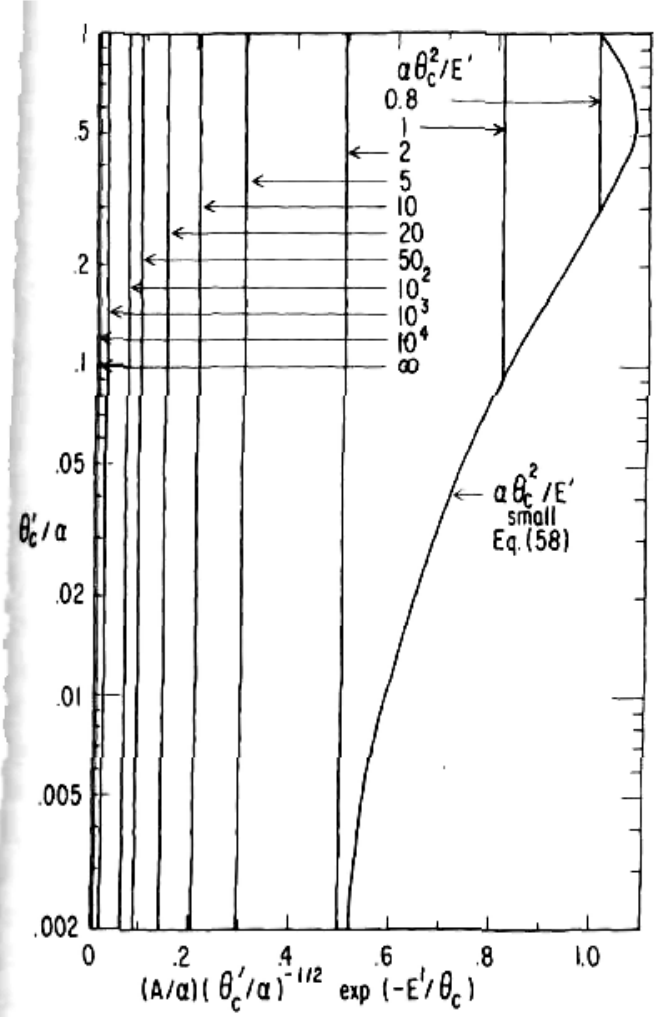

Fig. 4. Graph of ignition conditions for in-depth absorption with an arbitrary flux-time history.

of $\theta_{c}^{\prime} / \alpha$, as a function of

$$
(A / \alpha)\left(\theta_{c}^{\prime} / \alpha\right)^{-1 / 2} \exp \left(-E^{\prime} / \theta_{c}\right)
$$

for various values of $\alpha \theta_{c}^{2} / E^{\prime}$, as obtained from Eqs. 57 and 58, is shown in Fig. 4. To use Fig. 4, one must first solve his particular inert heatconduction problem to obtain $\theta_{c}$ and $\theta_{c}^{\prime}$ as a function of $\tau_{c}$.

It is remarkable that only the heat flux at the time of ignition (through the nondimensionalization), the inert surface temperature at the time of ignition, and the inert time rate of increase of the surface temperature at the time of ignition affect the ignition conditions of any given solid material. Many of the details of the history of inert heating are unimportant. However, in general, it is not possible to eliminate any of the three essential variables stated here in terms of other quantities. For example, one cannot ignore the inert time rate of increase of surface temperature at the time of ignition and consider instead the total amount of heat absorbed by the solid up until the time of ignition, as some authors have attempted to do (see some studies reviewed in Ref. 9).

If there is a distribution of the absorption coefficient over the frequency $\nu$ of the incident radiation, then in a fairly obvious notation the radiant heating term in Eq. 1 becomes

$$
\int_{0}^{\infty} I_{\nu} \alpha_{\nu} \exp \left(-\alpha_{\nu} \xi\right) d \nu
$$

This modification will require new solutions to be obtained for the inert-heating problem, although these solutions can be constructed by superposition, since with $\theta_{I} \equiv 1+\int_{0}^{\infty} \theta_{\nu} d \nu$, each $\theta_{\nu}$ separately satisfies Eq. 6 with

$$
\theta_{\nu}(\xi, 0)=\theta_{\nu}(\infty, \tau)=\theta_{\nu \xi}(0 ; \tau)=0
$$

The analysis of the transition stage will depend on whether the incident radiation corresponds principally to $\alpha_{\nu}=O\left(E^{\prime}\right)$ or to $\alpha_{\nu}=O(1)$. The transition stage that develops will be the one that produces the shortest ignition time. Since the ignition time for $\alpha_{\nu}=O\left(E^{\prime}\right)$ is appreciably less than that for $\alpha_{\nu}=O(1)$, ignition will occur in the regime of Section 5 , unless only a small fraction of the incident energy falls within that regime. A generalization of our analysis of Section 5 shows that in this case Eq. 57 is again obtained, provided that the more general definition

$$
\begin{aligned}
G & \equiv \int_{0}^{\infty} \exp \left\{-\int_{0}^{\infty} I_{\nu_{c}}[x\right. \\
& \left.\left.-\alpha_{1 \nu}^{-1} \exp \left(-\alpha_{1 \nu} x\right)\right] d \nu\right\} d x
\end{aligned}
$$

is used for $G$. In the lowest approximation, Eq. 57 then reveals that the factor $G$ can be ignored, provided that only the portion of the incident energy flux corresponding to the regime $\alpha_{\nu}=O\left(E^{\prime}\right)$ is employed in the $\dot{q}$ that is used to form the nondimensional time which appears in $A$ and in $\theta_{c}^{\prime}$. 
If almost all of the incident flux corresponds to $\alpha_{\nu}=O(1)$, then ignition occurs in the regime of Section 6, and it can be shown that Eq. 58 remains applicable, with $c_{0}$ related through Fig. 2 to the generalized quantity

$$
F \equiv\left(\frac{\int_{0}^{\infty} I_{\nu_{c}} \alpha_{\nu} d \nu-\theta_{c}^{\prime}}{2 \theta_{c}^{\prime}}\right),
$$

where the integral must exclude those values of $\nu$ for which $\alpha_{\nu}$ is of order $E^{\prime}$ or larger. After solving the inert-heating problem, one should calculate two ignition times, corresponding respectively to Eqs. 57 and 58 with the generalized definitions of $F$ and $G$, and select the shorter time, to complete the computation in the presence of a frequencydependent absorbtivity.

It is of interest to note from the analyses leading to Eqs. 57 and 58 that for solids of practical dimensions, the results given here are independent of the assumption of one-dimensional heat flow. The only requirement is that the narrow surface layer in which reaction first occurs must be approximated well as being one-dimensional. For the inert stage, a three-dimensional transient heatconduction problem must be solved. From this solution, for each point on the solid surface, the results given in this section can be applied to calculate ignition conditions. The shortest ignition time so obtained will represent the ignition time for the three-dimensional solid. The degree of generality that can be achieved with ease through asymptotic methods is impressive.

These results can be used to suggest an improved experimental technique for radiant ignition studies. The heat flux at the time of ignition and the history of surface temperature at the point of ignition are both amenable to measurement. From such measurements, $\theta_{c}$ and $\theta_{c}^{\prime}$ can be obtained, and Fig. 4 can then be used to calculate chemical rate constants from a few data points, provided, of course, that ignition occurs through a condensedphase reaction.

\section{Appendix: Variable Heat Flux}

To amplify the observations of Section 8 in more explicit terms, we first note that one can write
$I(\tau)=J\left(\alpha^{2} \tau\right)$, and that this factor will then appear in the last term of Eq. 6. It then becomes clear that we can write the solution for $\theta_{l}$ in the form given by Eq. 8 , but the function $f(u, v)$ will depend on the function $J(v)$ and will no longer be given by Eq. 9. It follows that Eqs. 11-14 will no longer be valid. However, it will still be possible to expand $\theta_{I}(0, \tau)$ about $\tau_{c}$, obtaining

$$
\theta_{l}(0, \tau)=\theta_{c}+\theta_{c}^{\prime}\left(\tau-\tau_{c}\right),
$$

where $\theta_{c}^{\prime} \equiv \theta_{I \tau}\left(0, \tau_{c}\right)$. We shall see that the $\xi$ dependence of the expansion of $\theta_{l}$ about $\xi=0$, needed in the transition-stage analysis, can be expressed in terms of $\theta_{c}$ and $\theta_{c}^{\prime}$. Therefore formulas for ignition conditions can be obtained in terms of the two parameters $\theta_{c}$ and $\theta_{c}^{\prime}$, which then must be expressed in terms of $\tau_{c}$ from an inert-heating analysis to obtain the ignition time.

Consider first the case analyzed in Section 5. In Eq. 18, the factor $\left(\pi \tau_{c}\right)^{-1 / 2}$ must be replaced by $\theta_{c}^{\prime}$, which is assumed to be of order unity. In Eqs. 20 and $22,\left(\pi \tau_{c}\right)^{1 / 4}$ is replaced by $\theta_{c}^{-1 / 2}$. Within the context of the asymptotic analysis, ignition cannot occur with negative values of $\theta_{c}^{\prime}$; if, for example, the flux is discontinued before ignition occurs, so that the surface temperature begins to decrease, then ignition never will occur. With $x$ and $\sigma$ as independent variables, the time-derivative terms in Eq. 1 and in Eq. 6 are of higher order than the space-derivative terms, and to lowest order Eq. 6 becomes

$$
\theta_{I x \boldsymbol{x}} \delta^{-2}=-\alpha_{1} J \exp \left(-\alpha_{1} x\right),
$$

whose solution is seen to be given by Eq. 26, when use is made of Eq. (A1) and the definition $J\left(\alpha^{2} \tau_{c}\right)=1$. Thus, Eqs. 28-32 still follow, with $\left(\pi \tau_{c}\right)^{1 / 4}$ replaced by $\theta_{c}^{\prime-1 / 2}$. The problem ex. pressed by Eq. 34 is again obtained, and the final solution, corresponding to Eq. 36, is given in Eq. 57.

Consider next the case analyzed in Section 6. When the modified form of Eq. 6 is solved for $\theta_{l \xi \xi}\left(0, \tau_{c}\right)$, and use is made of Eq. A1, then it is found that Eq. 11 becomes

$$
\theta_{I}=\theta_{c}+\theta_{c}^{\prime}\left(\tau-\tau_{c}\right)+\frac{\left(\theta_{c}^{\prime}-\alpha\right) \xi^{2}}{2} .
$$


In Eqs. 39, 41, and 42, we replace $\alpha g_{c}$ by $\theta_{c}^{\prime}$, and from Eq. A3 we then obtain Eq. 45, with

$$
F \equiv \frac{\left(\alpha-\theta_{c}^{\prime}\right)}{2 \theta_{c}^{\prime}}
$$

From Eq. A4 it might appear that a new condition for the generalized analysis to be valid is $\alpha>\theta_{c}^{\prime}$, but in fact this condition is assured by the inert-heating problem. The remainder of the analysis of Section 6 proceeds unchanged, and the generalization of Eq. 51 becomes Eq. 58 .

The work of the first author was partially supported by the Air Force Office of Scientific Research, through the European Office of the Office of Aerospace Research, United States Air Force, under Contract No. F61052-69-C-0036. The second author wishes to acknowledge fellowship support from the John Simon Guggenheim Memorial Foundation and additional support from the Air Force Office of Scientific Research, Office of Aerospace Research, United States Air Force under Project THEMIS Contract F44620-68-C-0010. We wish to thank Mr. V. Torroglosa for carrying out the computer calculation of $\mathrm{c}_{0}$.

\section{References}

1. Liñán, A., and Williams, F. A., Combustion Science and Technology 3, 91 (1971).

2. Ohlemiller, T. J., and Summerfield, M., "Radiative Ignition of Polymeric Fuels in an Oxidizing Gas," AFOSR Scientific Rept. No. AFOSR 69-2147TR (1969); Ohlemiller, T. J., Ph.D. Thesis, Princeton University (1969, unpublished).

3. Ohlemiller, T. J., and Summerfield, M., AIAA J. 6, 878 (1968).

4. Evans, M. W., Beyer, R. B., and MeCulley, L., J. Chem. Phys. 40, 2431 (1964).

5. Wise, H., Inami, S. H., and McCulley, L., Combustion and Flame 11, 483 (1967).

6. Boehringer, J. C., and Spindler, R. J., $A I A A J$. 1, 84 (1963).

7. Abramovitz, M., and Stegun, I. A., Handbook of Mathematical Functions, Dover, New York (1964), p. 261.

8. Bradley, H. H., Jr., Combustion Science and Technology 2, 11 (1970).

9. Merzhanov, A. G., and Averson, A. E., Combustion and Flame 16, 89 (1971).

(Received May 1971; revised version received A ugust 1971) 